\title{
Evaluating strategies to enhance the anti-tumor immune response to a carbohydrate mimetic peptide vaccine
}

\author{
BEHJATOLAH MONZAVI-KARBASSI, ANASTAS PASHOV, FARIBA JOUSHEGHANY, \\ CECILE ARTAUD and THOMAS KIEBER-EMMONS
}

\author{
Arkansas Cancer Research Center and Department of Pathology, \\ University of Arkansas for Medical Sciences, Little Rock, AR 72205, USA
}

Received January 9, 2006; Accepted February 24, 2006

\begin{abstract}
Carbohydrate mimetic peptides of tumor associated carbohydrate antigens (TACA) are T-cell-dependent antigens and, therefore, immunization with these surrogates is predicted to overcome the low immunogenicity of carbohydrate antigens. Consistent with this hypothesis, we show that among the potential immune cells involved, peptide immunization led to an increase in T-cell populations. While peptide mimetics may also function as TLR binding ligands, we did not observe evidence of involvement of NK cells. Examining tumor challenged animals, we observed that peptide immunization and not tumor cells rendered IL-12 responsiveness to T-cells, as T-cells from peptide-immunized mice produced IFN- $\gamma$ upon stimulation with IL-12. Cyclophosphamide administration enhanced the anti-tumor efficacy of the vaccine, which was achieved by enhancing T-cell responses with no effect on NK cell population. Prophylactic immunization of mice with a DNA construct encoding carbohydrate mimetic peptides indicated a specific role for the mimotope vaccine in antitumor immune responses. These data suggest a role for both $\mathrm{CD}^{+}$and $\mathrm{CD}^{+}$T-cells induced by mimotopes of TACA in protective immunity against tumor cells.
\end{abstract}

\section{Introduction}

Carbohydrates are the most abundantly expressed self-antigens on tumor cells and consequently they are perceived as viable targets for immunotherapy. Because tumor associated carbohydrate antigens (TACA) are T-cell-independent antigens and self-antigens, conjugation to immunologic carrier protein is essential to recruit T-cell help for antibody generation. Representative examples of these carbohydrate-based conjugate

Correspondence to: Dr Thomas Kieber-Emmons, Arkansas Cancer Research Center, University of Arkansas for Medical Sciences, 4301 West Markham St. Slot \#824, Little Rock, AR 72205, USA

E-mail: tke@uams.edu

Key words: tumor immunity, T-cells, carbohydrate, mimotope, peptide vaccines in clinical development include those directed toward gangliosides (1-3), polysialic acid (4), Globo-H (5), Lewis Y (LeY) (6), and the sialyl-Tn antigen (7). Conjugation of TACA does not, however, ensure an increase in immunogenicity because conjugation strategies do not uniformly enhance carbohydrate immunogenicity $(8,9)$. Furthermore, even with conjugation, the lack of induction of cellular immune responses that would amplify TACA-reactive humoral responses necessitates constant boosting with vaccine.

To overcome unresponsiveness to TACAs, we have focused our investigation on developing carbohydrate mimetic peptides (mimotopes) of TACAs as immunogens. Protein surrogates of TACAs are T-cell-dependent antigens and, therefore, immunization with these surrogates is predicted to facilitate cellular responses. Clinical characterization of anti-idiotypic antibodies that mimic the GD3 ganglioside antigen (10) and GD2 (11) have been described. The characterization of peptide mimotopes is at present limited to preclinical studies. Peptide mimotopes have been described for GD2 (12-14), GD3 (15), sialylated Lewis a/x (16), and LeY $(12,17)$. Peptide mimotopes of tumor antigenic epitopes potentially represent a novel vaccine approach to induce a tumor antigen-specific humoral and cellular response (18-22). Mimotopes function as xenoantigens and, consequently, can overcome tolerance to carbohydrate self-antigens. Unlike carbohydrate antigens and carbohydrate-conjugate vaccines, we have shown that mimotopes prime B- and T-cells for subsequent memory of carbohydrate antigens, facilitating long-term surveillance through recall of carbohydrate immune responses (23). This effect is a major advantage that would minimize the need for constant boosting. In addition, they can functionally emulate similar structures of TACA, inducing antibodies that recognize multiple TACAs and, therefore, functioning like a TACA multivalent vaccine (24).

Immune suppression in late stages is observed in many cancer patients and in model tumor disease, including methylcholanthrene (Meth A)-induced tumors in mice $(25,26)$. Secretion of suppressive cytokines, contact inhibition of CTL by tumor cells and the action of T regulatory cells (Tregs), NK and macrophages have been discussed as possible mechanisms of this immune dysfunction $(25,27-29)$. We have shown that therapeutic effectiveness of a mimotope-based vaccine depends on the tumor stage and time of vaccination (30). We speculated that suppression of the immune system 
and larger tumor size at the later stages are main causes of the failure of late vaccine administration. Immune suppression makes the redirection of an effective immune response with adequate strength to tumor antigens unlikely. It is shown that administration of cyclophosphamide (CY) at a low dose temporarily inhibits Treg cell function (31), which might lend to augment T-cell responses. Therefore, it is of clinical interest to investigate the potential of $\mathrm{CY}$ treatment during a therapeutic vaccine approach.

Here, we explore the effect of different immunotherapeutic regimens in order to maximize the tumor-protective immunity directed to carbohydrate antigens. We show that mimotope encoded DNA vaccine and alternative peptide formulations may prove effective in augmenting cellular responses despite the observed T-cell lymphopenia in Meth A tumor-bearing mice and that mimotope immunization is necessary for rendering IL-12 responsiveness and the tumor's infiltration ability to the T-cells of these animals. In particular, we demonstrate that CY treatment might improve the therapeutic outcome of a carbohydrate mimotope vaccine.

\section{Materials and methods}

Mice and tumor inoculation. Six- to eight-week-old BALB/c female mice were purchased from The Jackson Laboratory (Bar Harbour, ME). To establish tumors, each mouse was inoculated subcutaneously into the right flank with $5 \times 10^{5}$ Meth A cells (methylcholanthrene-induced sarcoma of BALB/c origin) (17). Tumor growth was measured using a caliper and was recorded as the mean of two orthogonal diameters $[(a+b) / 2]$.

Immunization. As in our previous studies $(17,32)$, peptide 106 (sequence, GGIYWRYDIYWRYDIYWRYD) was synthesized as a monomer, a multiple antigen peptide (MAP; Research Genetics, Huntsville, AL) or conjugated to a protein transduction domain, GGAYARAAARQARA (106PTD) (33). Each mouse received $100 \mu \mathrm{g}$ of 106MAP and $20 \mu \mathrm{g}$ of QS-21 (Antigenics Inc., Framingham, MA) intraperitoneally (i.p.), both resuspended in $100 \mu 1$ of PBS three times at 5-day intervals. Recombinant murine IL-12 (Sigma, St. Louis, MO) was administered i.p. once daily for 5 days, starting on the day of the last peptide immunization. For the construction of expression vectors coding for peptides 106 and 911 (YRY RYGRYRSGSYRYRYGRYRSGS), oligonucleotides were synthesized and inserted into pcDNA3 vector (Invitrogen, San Diego, CA) fused in frame to an oligonucleotide coding for the adenovirus E3 leader sequence as described earlier (34). Cloning was confirmed by DNA sequencing.

Flow cytometry and antibodies. Anti-mouse CD4 (GK1.5), CD8 (SF1-1.1), CD3 ${ }^{+}$(17A2) and Pan-NK cells (DX5) and their isotypes were purchased from BD-PharMingen (San Diego, CA). Cells were resuspended in a buffer containing, Dulbecco's phosphate-buffered saline (DPBS, Life Technologies), $1 \%$ BSA and $0.1 \%$ sodium azide (FACS buffer), and incubated with conjugated antibodies $(10 \mu \mathrm{g} / \mathrm{ml})$ for $30 \mathrm{~min}$ on ice. Acquisition and analysis were performed as described elsewhere. Cells stained with isotypes were used as negative controls.
T-cell purification. Splenocytes were harvested from spleens and prepared by lysis of erythrocytes and consequent washing several times with fresh media (32). Splenocytes were first passed through nylon wool and then, using MiniMACS (Miltenyi Biotec, Auburn, CA), NK cells were depleted using anti-NK cell (DX5) microbeads. Finally, T-cells were positively purified by Thy1.2-coated beads. Purified T-cells were tested for purity as $>97 \%$ positive for anti-CD3 antibody.

IFN- $\gamma$ production by purified T-cells. Purified T-cells (1x10\% $\mathrm{ml}$ ) were cultured in 96- or 24-well plates with various doses of rIL-12. After $48 \mathrm{~h}$ of stimulation, supernatant was harvested and stored at $-20^{\circ} \mathrm{C}$ until use. The concentration of IFN- $\gamma$ was measured using a quantitative ELISA kit (BioSource International Inc., Camarillo, CA) according to the manufacturer's instructions.

Proliferation assay. Splenocytes from mice immunized with 106PTD and 106MAP were stimulated in vitro with 106, 106MAP, 106PTD or peptide 12H (KVWQMYWPSGQP) selected for binding to monoclonal antibody $2 \mathrm{G} 12$ as an irrelevant peptide. The splenocytes were plated at $10^{5}$ cells per well in U-bottom 96-well plates in the presence of serial dilutions of the tested peptides in Dulbecco's modified Eagle's medium (DMEM), supplemented with $10 \%$ fetal calf serum, $0.2 \mathrm{mM}$ 2-mercaptoethanol, L-glutamine, sodium pyruvate, penicillin/streptomycin and non-essential amino acids. After 7 days, the proliferation was measured by BrDU incorporation using colorimetric cell proliferation ELISA kit (Roche Diagnostics GmbH, Mannheim).

Confocal microscopy. The uptake of 106PTD peptide in living cells was observed after treating suspension of mouse mammary tumor cell line 4T1 (ATCC, CRL-2539) with 106PTD-FITC conjugate in the presence of propidium iodide to exclude dead cells. A Zeiss LSM410 confocal microscope (Carl Zeiss Ltd.) was used and 106PTD-FITC and PI were visualized using standard FITC and PI filter sets. Images of the same field were acquired in transmitted light and fluorescence at 515 and $617 \mathrm{~nm}$.

Surface plasmon resonance. The ability of peptides to translocate through a bilipid layer was tested using Biacore $\mathrm{X}$ and a hydrophobic L1 biosensor chip (Biacore AB, Uppsala, Sweden). The liposome suspension was prepared by drying, in a rotary evaporator, $4 \mathrm{ml}$ of $7.5 \mathrm{mM}$ solution of dimyristoyl phosphatidyl choline (DMPC) in 1:1 mixture of chloroform and methanol followed by addition of $60 \mathrm{ml}$ of distilled water to the flask and sonication for $15 \mathrm{~min}$ at $37^{\circ} \mathrm{C}$. The resulting suspension was cleared by centrifugation for $10 \mathrm{~min}$ at $4000 \mathrm{x}$. The surface of an L1 chip was conditioned by 5-min injection with $40 \mathrm{mM}$ n-Octyl $\beta$-D-glucopyranoside followed immediately by injection with the liposome suspension at $2 \mu \mathrm{l} /$ $\min$. The liposome layer was considered continuous when the sensogram reached a plateau. Different peptides were passed over the liposome surface dissolved in HBS-N nuffer (Biacore $\mathrm{AB})$ at $10 \mu \mathrm{M}$ and a $10 \mu \mathrm{l} / \mathrm{min}$ flow rate. The incorporation was characterized by high uptake of the peptide by the bilipid layer with an extremely low dissociation rate. 
A

CD4+ cells

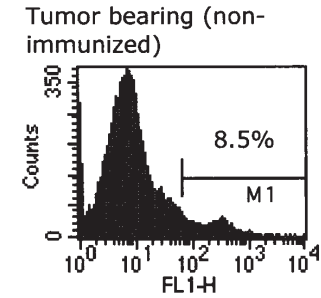

Cured (immunized)

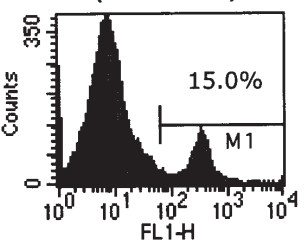

CD8+ cells
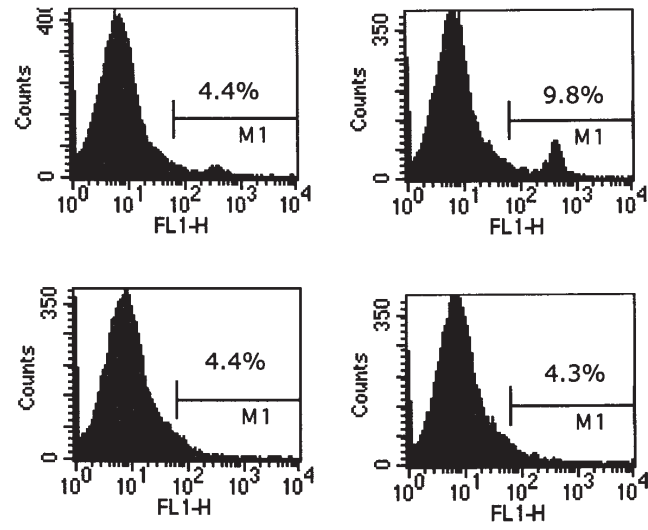

B

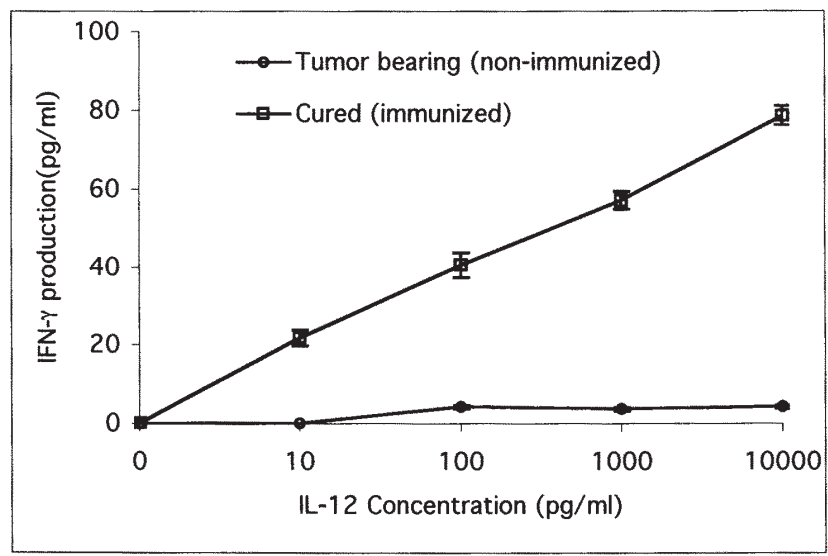

Figure 1. Immunization with peptide 106 augmented the proportion of $\mathrm{CD}^{+}$ and $\mathrm{CD} 8^{+}$cells among splenocytes and rendered IL-12 responsiveness among T-cells. (A) Tumor-bearing (non-immunized) and cured (peptide immunized/IL-12-treated) mice were sacrificed and splenocytes were collected and deleted from erythrocytes. Freshly prepared splenocytes were stained with Pan-NK cell marker (DX5) and anti-CD4, -CD8 FITCconjugated mAbs. The results are representative of two experiments. Negative controls were established using isotype controls. Percentage of positive cells for each histogram is shown. (B) Tumor-bearing (nonimmunized) and cured (immunized/IL-12-treated) mice were sacrificed, splenocytes were collected and T-cells were purified. Purified T-cells were incubated with serial dilutions of rIL-12 for $48 \mathrm{~h}$. Then supernatants were collected and the concentration of IFN- $\gamma$ was determined. The results are representative of three independent experiments.

Statistical analysis. Experiments were repeated at least two times. Statistical analyses were performed using Student's t-test and $\mathrm{P}<0.05$ was regarded as statistically significant.

\section{Results}

Mimotope immunization led to an increase in T-cell populations and IL-12 responsiveness of T-cells. We have shown that peptide 106 elicits anti-tumor CTLs that, in the presence of IL-12, lead to a complete eradication of tumors $(30,32)$. To further explore the effect of mimotope immunization on the immune cell population and homeostasis in tumor-bearing mice, we analyzed the percentage of T-cells and NK cells in splenocytes collected from non-treated tumor-bearing mice and mice cured of their tumors after immunization. We examined the expression of CD4, CD8 and Pan-NK cell marker on the surface of freshly isolated splenocytes (Fig. 1). Representative littermates from the nonimmunized tumor-bearing group showed lower than normal (35-37) percentages of T-cells; however, the percentage of $\mathrm{CD}^{+}{ }^{+}$and $\mathrm{CD}^{+}{ }^{+}$T-cells almost doubled, $15 \%$ vs $8.5 \%$ for $\mathrm{CD}^{+}$and $9.8 \%$ vs $4.4 \%$ for $\mathrm{CD}^{+}$cells, in mice cured of their tumor upon peptide/IL-12 therapy. We did not detect any difference between groups of mice regarding NK cell population using the DX5 antibody. We observed that eradication of tumor mass upon peptide immunization was associated with increased frequency of splenic T-cells. These data suggest that peptide immunization induced a reversal of the depression of T-cell homeostasis leading to 'immunological excision' of the tumor.

We have observed that IL-12 administration during and after peptide immunization dramatically improved the outcome of therapy (30) but, unlike other tumor models, Meth A fibrosarcomas are insensitive to systemically administered IL-12 treatment (38). To further explore the dependency of the IL-12 effect on peptide immunization, an IL-12 responsiveness test was performed on the T-cell populations of tumorbearing mice and those cured of their tumor upon peptide immunization (Fig. 1B). Mice from non-immunized/tumorbearing, and immunized/cured groups were sacrificed and their T-cells were purified using nylon wool followed by depletion of NK cells and subsequent positive selection for Thy 1.2 using MACS. The purity of the isolated T-cells was $>97 \%$ as assessed by expression of CD3 molecule by FACS. Purified T-cells were seeded and stimulated with various concentrations of IL-12 and induction of IL-12 responsiveness was determined by measuring IFN- $\gamma$ production. T-cells from peptide-immunized animals produced IFN- $\gamma$ upon stimulation with IL-12 (Fig. 1B). Since we have reported observing T-cell infiltration into tumor tissue (30), our present results suggest that mimotope vaccination amplifies a population of T-cells able to infiltrate into Meth A tumor mass. IL-12 administration stimulates the expansion of such infiltrating T-cells, leading to efficient elimination of the tumor mass.

\section{Cyclophosphamide administration enhanced mimotope} induced T-cell population. We consider our tumor-clearing immunization as a prime/boost regimen, where a cell-primed immune system is being boosted with the peptide. At the same time, the peptide/IL-12 formulation seems to unleash an existing immune response, which was suppressed by tumor-induced elements prior to immunization. It has been shown that immunization of mice with Meth A cells rendered IL-12 responsiveness to T-cells (39). To determine whether initial priming with tumor cells improves the outcome of peptide immunization, the first peptide immunization of our standard approach (30) was replaced with inactivated Meth Acell immunization. Tumor-bearing mice, 7 days after tumor inoculation, were initially immunized with attenuated cells 

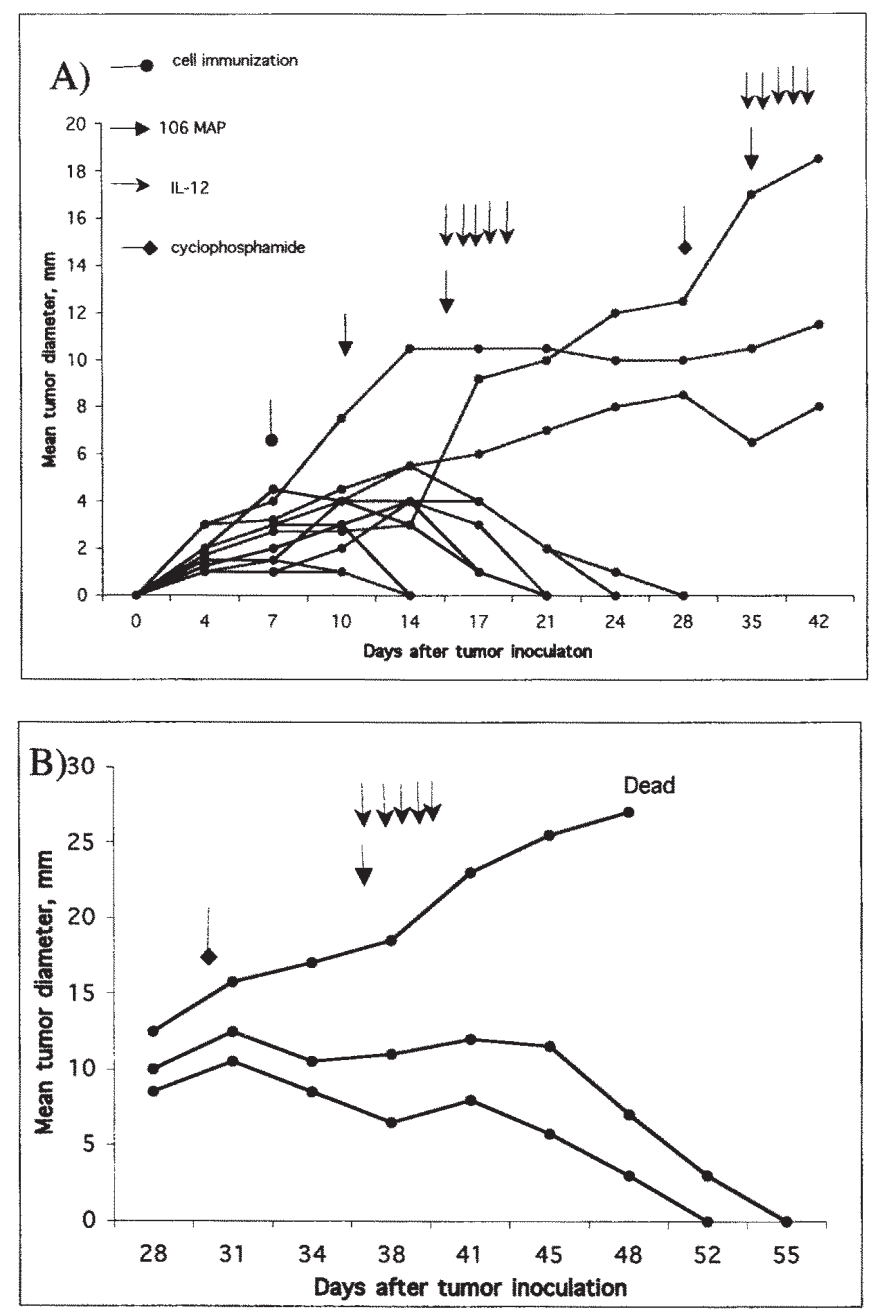

C)

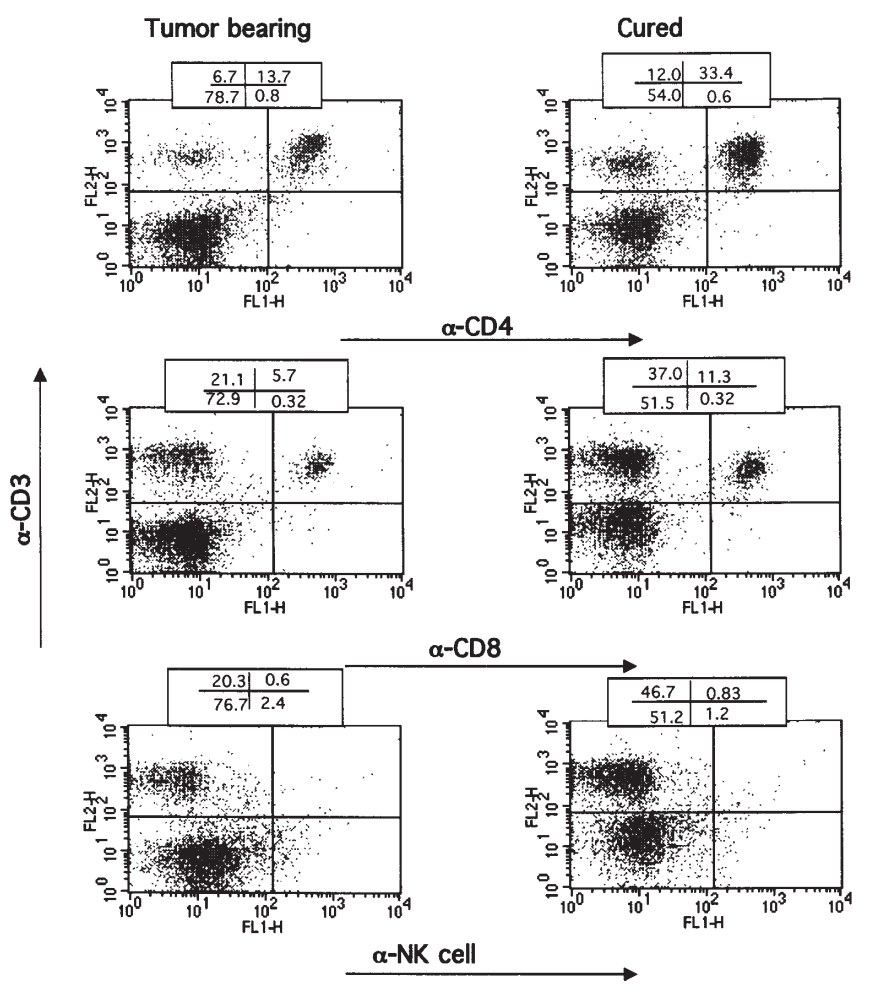

Figure 2. Effect of priming with cells on Meth A tumor growth and regression. (A) BALB/c female mice were inoculated s.c. with $5 \times 10^{5}$ Meth A cells on day 0 . Seven days after tumor inoculation mice were immunized i.p. with mitomycin C-inactivated Meth A cells/QS-21 (107 cells/mouse i.p.). The immunization regimen was followed by $2 \times 106 \mathrm{MAP} / \mathrm{QS}-21$ immunization at $4-5$ day intervals. IL- 12 was administered following peptide immunization at $500 \mu \mathrm{g} / \mathrm{mouse}$ daily for 5 days, starting on the day of the last peptide immunization. Tumor growth is expressed as the mean diameter for each individual mouse. (B) Mice with growing tumors were treated with cyclophosphamide $(2.5 \mathrm{mg} /$ mouse $)$ and re-immunized as indicated. One mouse was euthanized and considered experimentally dead because of the size of tumor observing the animal protocol in use. (C) Treatment with CY and IL-12 mediated an increase in CD4+ and $\mathrm{CD}^{+}$cells. Immunized tumor-bearing mice (uncured) and a mouse that underwent Cy and IL-12 treatment after eradication of the tumor were sacrificed and freshly prepared $\mathrm{CD}^{+}$splenic lymphocytes were stained with anti-CD4,-CD8 and -NK cell marker. The results are representative of two experiments. Cells stained with isotypes were used as negative controls. The percentage of cells for each quadrant is shown.

$\left(10^{7} /\right.$ mouse, i.p.) and then with a combination of peptide/IL-12 (Fig. 2A). This immunization regimen was less efficient as we recorded 3 immune mice out of 12 (Fig. 2A). The results suggest that, for achieving high anti-tumor efficacy, early peptide immunization is necessary and tumor clearance depends on the time of the first peptide immunization and the size of the tumor at that time. As shown by another group working with this cell line, cell immunization is not enough to generate a therapeutic immunity to tumor cells despite rendering IL-12 responsiveness to T-cells (39). This means, that induction of IL-12 responsiveness is required for the generation of efficient anti-tumor cellular responses but it is not enough to generate therapeutic immunity against tumors. Lack of T-cell migration into tumor mass was considered as the main reason for the failure of a cell-based immunization therapy (39).

Since the overriding of tolerance mechanisms seemed at least as important as boosting the anti-tumor CTL response, we tried to further stimulate the eradication of tumors in immunized/tumor-bearing animals (escaped mice) by treating them with CY at a dose that has been shown to suppress the Treg cell function (31) (Fig. 2A and B). CY injected littermates were re-immunized with a single peptide dose after 7 days, followed by 5 doses of IL-12. We speculated that by using this regimen, we might expand the repertoire of effector T-cells, which may help in eradicating tumors of larger size. Following CY and peptide/IL-12 therapy, tumor regression was marked in 2 animals out of 3 (Fig. 2B). Splenocytes were isolated and stained with cell surface markers by flow cytometry (Fig. 2C). Splenic $\mathrm{CD}^{+}$and $\mathrm{CD}^{+} \mathrm{T}$-cells were increased in cured mice as compared with the mouse whose tumor continued to grow. No difference in NK cell population was observed between responders and non-responders.

Vaccination with peptide 106 encoded DNA inhibited tumor growth. To further understand the specificity of the peptide and 

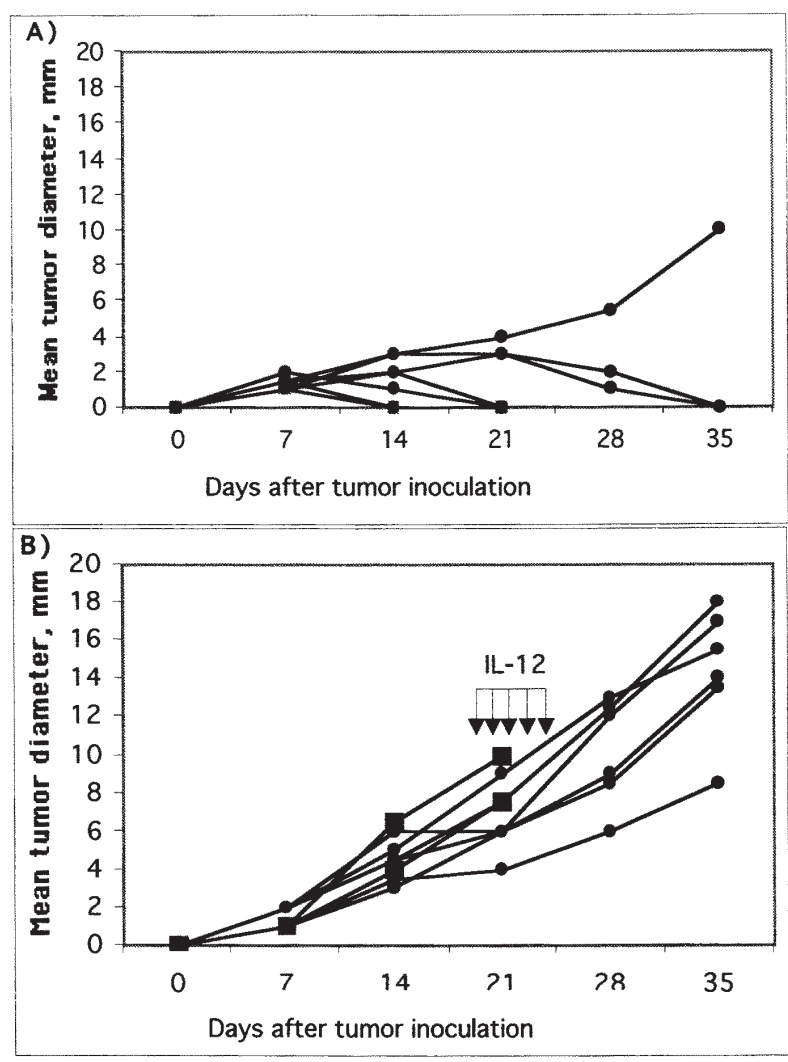

Figure 3. Vaccination with DNA format of the peptide suppressed tumor growth in further challenging the immunized mice with Meth A cells. Mice were immunized with DNA constructs of 106 (A) and 911 (B) peptides twice at a 3-week interval. Four months after the boost mice were challenged with $5 \times 10^{5}$ Meth A cells s.c. Tumor growth is expressed for each individual mouse. Squares show individual mice sacrificed because of their large tumor size before the end of experiment.

the possible role of epitope spreading effects, we compared peptides 911 and 106 in a prophylactic DNA immunization regimen (Fig. 3). We have shown previously that peptide 911 activates T-cells (24). Groups of mice were immunized with respective DNA constructs twice, rested for 4 months and then challenged with Meth A tumor cells (Fig. 3). Tumor growth inhibition was observed in mice immunized with the peptide 106 encoded DNA construct (Fig. 3A) but not in mice immunized with the construct of peptide 911 (Fig. 3B). Addition of IL-12 did not affect the outcome of the 911 immunized mice (Fig. 3B). These data imply that specific T-cell activation against Meth A cells was induced by peptide 106 immunization that functions in the context of long-term memory.

Optimization of the multiple antigenic mimotope to be taken up by cells. In a previous study, we showed that MAP 106 stimulated a cellular response in splenic T-cells, which was inhibited by anti-MHC class II antibodies and depended on the presence of APC (32). At the same time, analysis of the sequence of peptide 106 with available algorithms identified an $\mathrm{H}-2 \mathrm{~K}^{\mathrm{d}}$ binding motif but not a class II one. To further improve the immunogenicity of the peptide, the strategy of introducing a Th cell epitope and a protein transduction domain (PTD) by the same structure was developed in the present study. To this end, we synthesized a longer version of peptide 106 (GGIYWRYDIYWRYDIYWRYDGGAYARA AARQARA) which included the PTD sequence (AYARAA ARQARA) that coincidentally rendered the structure an I- $\mathrm{A}^{\mathrm{d}}$ binding motif. The ability of the 106/PTD peptide to penetrate the liposome layer was demonstrated by surface plasmon resonance using an L-type biosensor (Fig. 4A). 106PTD-FITC conjugate was co-incubated with mouse tumor cells 4T1 that showed cytoplasmic and nuclear fluorescence indicating efficient protein transduction (Fig. 4B). Immunization with 106PTD and 106MAP elicited T-cell responses cross-reactive between these two peptides but not to monomeric 106, suggesting that the addition of PTD improved the immunogenicity of peptide 106 and that recognition depends on the form of presentation of the peptide (Fig. 4C).

\section{Discussion}

In previous publications, we have established that immunization with peptide 106 stimulates T-cell activation triggering IFN- $\gamma$ release, suggesting that peptide immunization with QS-21 is able to polarize the Th1 subset $(32,40)$. We have shown that, while Meth A cell priming and boosting can lead to CTL activity against Meth A cells, peptide boosting increased the level of cytotoxicity against Meth A cells to a statistically significant level as compared with cytotoxicity against P815 cells used as a control target, indicating a cross-reactive nature between peptide- and tumor-specific CTL responses (32). It has been shown that vaccination with inactivated Meth A cells had no effect on tumor growth, suggesting a superiority of the 106 peptide vaccine to a cell-based one in this model $(30,39)$. We have demonstrated that immunization with only peptide 106 is not sufficient for induction of a state of reliable immunity that can eradicate tumors. IL-12 is known to exhibit potent anti-tumor activity in a number of murine tumor models. Further treatment of peptide immunized mice with IL-12 considerably helped in stimulating the eradication of tumors. However, the Meth A sarcoma model proved refractory to immunization with inactivated cells, systemic IL-12 administration or a combination of both $(38,39)$.

Our data show that there is a proportional increase in $\mathrm{CD}^{+}$and $\mathrm{CD}^{+} \mathrm{T}$-cells among splenocytes of treated mice compared to tumor-bearing mice. We did not observe any differences between the splenocytes of these two groups of mice regarding the expression of Pan-NK cell marker as tested using DX5 antibody. As resting T-cells do not express the IL-12 receptor and IL-12 responsiveness is induced after TCR stimulation, purified T-cells were stimulated with IL-12 in vitro. The data indicate that, in tumor-bearing animals, T-cells were not sensitized with tumor antigens, while peptide immunization induced T-cell responsiveness to IL-12. Another group showed that immunization with inactivated Meth A cells induced IL-12 responsiveness in the T-cell population, but no anti-tumor effect was observed with such treatment (39). This proposes that, besides sensitizing T-cells to IL-12, peptide immunization could have additional effects on the effector phase of the T-cell response. We have shown lymphocytes infiltrating into tumor tissue upon peptide-vaccine/IL-12 administration (30). We conclude that, in addition to sensitizing T-cells to IL-12, peptide immunization may trigger tumor cross-reactive T-cells able to migrate into tumor mass. 
A)

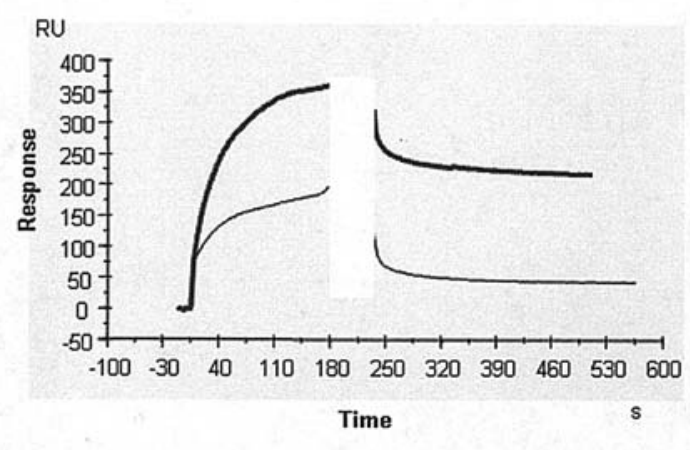

C)

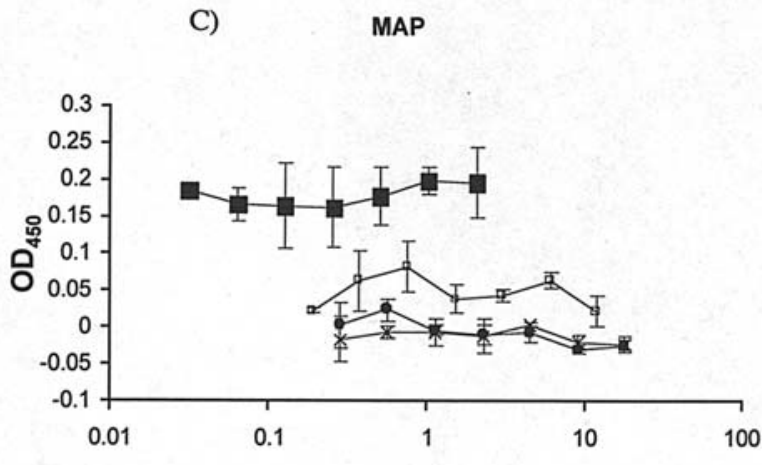

B)
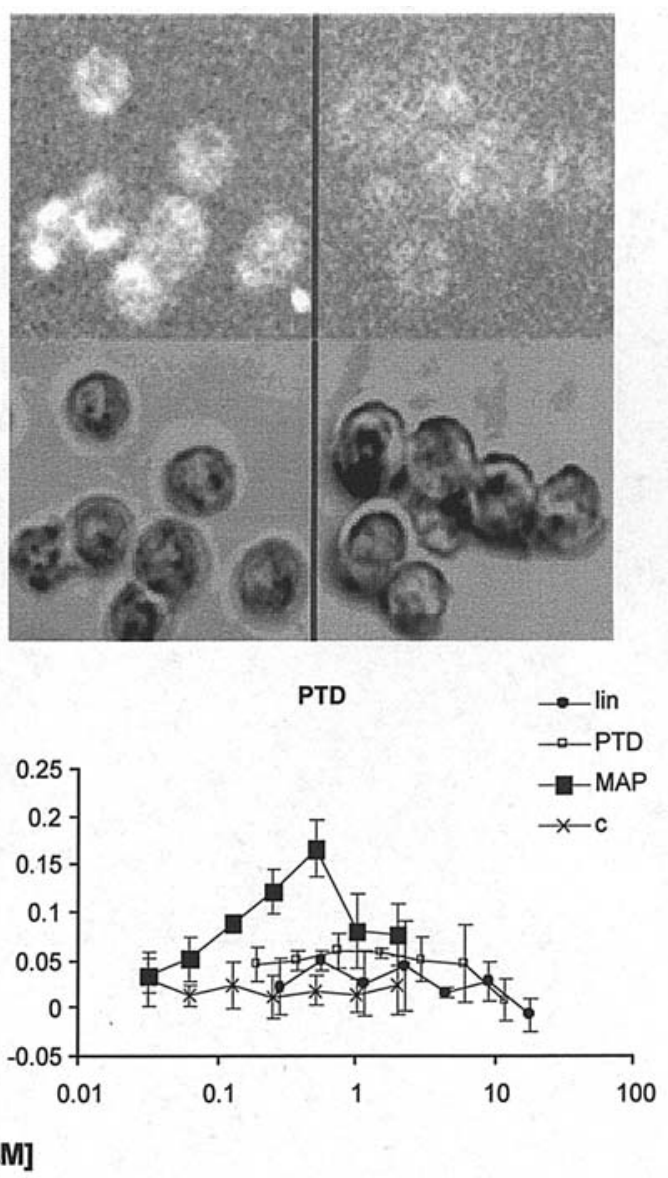

Figure 4. (A) Binding of 106 (thin line) and 106PTD (thick line) to liposomes coated on L1 chip, Biacore. The peptides were passed over the liposome surface in equal concentrations. The presence of the PTD increases the uptake of the peptide. (B) Uptake of 106PTD-FITC peptide by live (excluding PI) murine 4T1 cells - confocal microscopy (left) compared to unstained cells (right). The upper panel shows the fluorescent chanel. (C) Proliferation assay (BrDU cell ELISA) of splenocytes from BALB/c mice immunized with MAP (left) or PTD (right) versions of 106 peptide and stimulated in vitro with the same antigens as well as with monomer 106 and an irrelevant 12-mer MAP.

The data indicate that low-dose CY treatment during the vaccination period can improve the efficacy of the vaccine, which could be applicable to a later stage of tumor development. The mechanism may involve synergistic effects in the overriding of the immune tolerance to the tumor. Immunization with peptide 106 after treatment with CY increased the relative number of splenic T-cells, indicating a successful removal of the homeostatic depression on the T-cells. The immunostimulatory effect of low-dose CY in tumor immunotherapy has been intensely investigated (41-43). Recently, the CY effect has been shown to depend on the suppression of $\mathrm{CD}^{+}{ }^{+} \mathrm{CD} 25^{+}$Tregs $(31,44,45)$. We speculate that $\mathrm{CY}$ facilitates restoring the T-cell homeostasis through non-specific inhibition of the Tregs in the immunized mice. Tregs have been shown to control the homeostatic set point of the T-cell population and to play a central role in the tumor escape from the immune system (46-49). It has been shown that removal of Tregs can lead to the recruitment of higher avidity CTL in the anti-tumor response (50).

In our system, the combination of the specificity of the mimotope, the adjuvant activity of IL-12 and the immuneactivator effect of $\mathrm{CY}$ acted synergistically to induce effective cellular immune response to larger tumor masses at a later time point. Indeed, priming with inactivated Meth A cells did not improve on the immunogenicity of the following boost with 106, which failed at complete protection due to the late time point of the peptide immunization.

The fact that DNA immunization with the peptide 911 sequence did not mediate tumor growth inhibition, even with the addition of IL-12, implies that peptide 106 exclusively activates specific anti-Meth A tumor cellular responses. We have previously shown that immunization with peptide 106 induces cellular responses that are not achievable by immunization with carbohydrate alone (23).

Although cellular responses generated by the peptide mimotope may enhance CTL induction, vaccination with peptide alone appears not to be completely sufficient in the effector phase when challenged with a very high tumor burden. Our results are very narrow with regards to the breadth of carbohydrate directed cellular responses. Further efforts to optimize and isolate the carbohydrate moieties associated with presented glycopeptides may facilitate vaccine applications for eradication of metastatic lesions by both antibody mediated lysis and cellular responses. This possibility has yet to be proved with the appropriate models but suggests that, for certain carbohydrate antigens, carbohydrate mimetic peptides might augment cellular responses other than delayed-type hypersensitivity-like responses. 
Our results suggest that mimotopes are entering another phase in that they may be suitable to generate cellular responses to naturally processed glycopeptides providing an advantage over carbohydrate-based vaccines. The potential remains to further identify mimotopes and improve upon their immunogenicity to amplify functional carbohydrate directed immune responses against circulating or disseminated tumor cells to impact on cancer survival. Consequently, peptide mimotopes are effective immunogens that should be clinically evaluated for their ability to amplify carbohydrate immune responses against circulating or disseminated tumor cells.

We believe that this vaccine approach is particularly interesting for cancer prevention because: a) TACA are expressed very early during neoplastic transformation rendering possible immunosurveillance by CTL; b) the same TACA-based vaccine can be used in a variety of tumors, circumventing the limitation of epitope mapping; and c) by designing appropriate peptide backbone sequence(s), it may be possible to target multiple supertype MHC class I alleles, rendering feasible future CTL-based vaccines applicable on a large population scale.

\section{Acknowledgements}

This work was supported by grants from the US Army Breast Cancer Program (DAMD17-01-1-0366) and the NIH (CA-089480) to T.K.-E.

\section{References}

1. Guthmann MD, Bitton RJ, Carnero AJ, et al: Active specific immunotherapy of melanoma with a GM3 ganglioside-based vaccine: a report on safety and immunogenicity. J Immunother 27: 442-451, 2004

2. Krug LM, Ragupathi G, Hood C, et al: Vaccination of patients with small-cell lung cancer with synthetic fucosyl GM-1 conjugated to keyhole limpet hemocyanin. Clin Cancer Res 10: 6094-6100, 2004.

3. Ragupathi G, Livingston PO, Hood C, et al: Consistent antibody response against ganglioside GD2 induced in patients with melanoma by a GD2 lactone-keyhole limpet hemocyanin conjugate vaccine plus immunological adjuvant QS-21. Clin Cancer Res 9: 5214-5220, 2003.

4. Krug LM, Ragupathi G, Ng KK, et al: Vaccination of small cell lung cancer patients with polysialic acid or N-propionylated polysialic acid conjugated to keyhole limpet hemocyanin. Clin Cancer Res 10: 916-923, 2004.

5. Slovin SF, Ragupathi G, Fernandez C, et al: A bivalent conjugate vaccine in the treatment of biochemically relapsed prostate cancer: a study of glycosylated MUC-2-KLH and Globo H-KLH conjugate vaccines given with the new semi-synthetic saponin immunological adjuvant GPI-0100 OR QS-21. Vaccine 23: 3114-3122, 2005.

6. Sabbatini PJ, Kudryashov V, Ragupathi G, et al: Immunization of ovarian cancer patients with a synthetic Lewis(y)-protein conjugate vaccine: a phase 1 trial. Int J Cancer 87: 79-85, 2000.

7. Holmberg LA and Sandmaier BM: Vaccination with Theratope (STn-KLH) as treatment for breast cancer. Expert Rev Vaccines 3: 655-663, 2004

8. McCool TL, Harding CV, Greenspan NS and Schreiber JR: B- and T-cell immune responses to pneumococcal conjugate vaccines: divergence between carrier- and polysaccharide-specific immunogenicity. Infect Immun 67: 4862-4869, 1999.

9. Gilewski T, Ragupathi G, Bhuta S, et al: Immunization of metastatic breast cancer patients with a fully synthetic globo $\mathrm{H}$ conjugate: a phase I trial. Proc Natl Acad Sci USA 98: 3270-3275, 2001.

10. Chapman PB, Wu D, Ragupathi G, et al: Sequential immunization of melanoma patients with GD3 ganglioside vaccine and antiidiotypic monoclonal antibody that mimics GD3 ganglioside. Clin Cancer Res 10: 4717-4723, 2004.
11. Foon KA, Lutzky J, Baral RN, et al: Clinical and immune responses in advanced melanoma patients immunized with an anti-idiotype antibody mimicking disialoganglioside GD2. J Clin Oncol 18: 376-384, 2000.

12. Qiu J, Luo P, Wasmund K, Steplewski Z and Kieber-Emmons T: Towards the development of peptide mimotopes of carbohydrate antigens as cancer vaccines. Hybridoma 18: 103-112, 1999.

13. Bolesta E, Kowalczyk A, Wierzbicki A, et al: DNA vaccine expressing the mimotope of GD2 ganglioside induces protective GD2 cross-reactive antibody responses. Cancer Res 65: 3410-3418, 2005

14. Forster-Waldl E, Riemer AB, Dehof AK, et al: Isolation and structural analysis of peptide mimotopes for the disialoganglioside GD2, a neuroblastoma tumor antigen. Mol Immunol 42: 319-325, 2005 .

15. Willers J, Lucchese A, Kanduc D and Ferrone S: Molecular mimicry of phage displayed peptides mimicking GD3 ganglioside. Peptides 20: 1021-1026, 1999.

16. O I, Kieber-Emmons T, Otvos L Jr and Blaszczyk-Thurin M: Peptides mimicking sialyl-Lewis A isolated from a random peptide library and peptide array. Ann NY Acad Sci 886: 276-279, 1999.

17. Kieber-Emmons T, Luo P, Qiu J, et al: Vaccination with carbohydrate peptide mimotopes promotes anti-tumor responses. Nat Biotechnol 17: 660-665, 1999.

18. Hafner C, Wagner S, Jasinska J, et al: Epitope-specific antibody response to Mel-CAM induced by mimotope immunization. J Invest Dermatol 124: 125-131, 2005.

19. Hardy B and Raiter A: A mimotope peptide-based anti-cancer vaccine selected by BAT monoclonal antibody. Vaccine 23: 4283-4291, 2005

20. Chen Y, Wu K, Guo C, et al: A novel DNA vaccine containing four mimicry epitopes for gastric cancer. Cancer Biol Ther 4: 308-312, 2005.

21. Wagner S, Hafner C, Allwardt D, et al: Vaccination with a human high molecular weight melanoma-associated antigen mimotope induces a humoral response inhibiting melanoma cell growth in vitro. J Immunol 174: 976-982, 2005.

22. Tumenjargal S, Gellrich S, Linnemann T, et al: Anti-tumor immune responses and tumor regression induced with mimotopes of a tumor-associated T cell epitope. Eur J Immunol 33: 3175-3185, 2003.

23. Monzavi-Karbassi B, Shamloo S, Kieber-Emmons M, et al: Priming characteristics of peptide mimotopes of carbohydrate antigens. Vaccine 21: 753-760, 2003.

24. Cunto-Amesty G, Dam TK, Luo P, et al: Directing the immune response to carbohydrate antigens. J Biol Chem 276: 30490-30498, 2001

25. Ohm JE and Carbone DP: Immune dysfunction in cancer patients. Oncology 16: 11-18, 2002.

26. Kiessling R, Wasserman K, Horiguchi S, et al: Tumor-induced immune dysfunction. Cancer Immunol Immunother 48: 353-362, 1999.

27. Gabrilovich DI, Velders MP, Sotomayor EM and Kast WM: Mechanism of immune dysfunction in cancer mediated by immature Gr-1+ myeloid cells. J Immunol 166: 5398-5406, 2001.

28. Levey DL, Udono H, Heike M and Srivastava PK: Identification of a tumor-associated contact-dependent activity which reversibly downregulates cytolytic function of $\mathrm{CD}^{+} \mathrm{T}$ cells. Cancer Immun 1: 5,2001 .

29. Ohm JE and Carbone DP: VEGF as a mediator of tumorassociated immunodeficiency. Immunol Res 23: 263-272, 2001.

30. Monzavi-Karbassi B, Luo P, Jousheghany F, et al: A mimic of tumor rejection antigen-associated carbohydrates mediates an antitumor cellular response. Cancer Res 64: 2162-2166, 2004.

31. Lutsiak ME, Semnani RT, DePascalis R, Kashmiri SV, Schlom J and Sabzevari H: Inhibition of CD4(+)25+ T regulatory cell function implicated in enhanced immune response by low-dose cyclophosphamide. Blood 105: 2862-2868, 2005.

32. Monzavi-Karbassi B, Cunto-Amesty G, Luo P, Shamloo S, Blaszcyk-Thurin $\mathrm{M}$ and Kieber-Emmons T: Immunization with a carbohydrate mimicking peptide augments tumor-specific cellular responses. Int Immunol 13: 1361-1371, 2001

33. Ho A, Schwarze SR, Mermelstein SJ, Waksman G and Dowdy SF: Synthetic protein transduction domains: enhanced transduction potential in vitro and in vivo. Cancer Res 61: 474-477, 2001.

34. Kieber-Emmons T, Monzavi-Karbassi B, Wang B, Luo P and Weiner DB: Cutting edge: DNA immunization with minigenes of carbohydrate mimotopes induce functional anti-carbohydrate antibody response. J Immunol 165: 623-627, 2000. 
35. Pruett SB, Fan R, Myers LP, Wu WJ and Collier S: Quantitative analysis of the neuroendocrine-immune axis: linear modeling of the effects of exogenous corticosterone and restraint stress on lymphocyte subpopulations in the spleen and thymus in female B6C3F1 mice. Brain Behav Immun 14: 270-287, 2000.

36. Wei LX, Zhou JN, Roberts AI and Shi YF: Lymphocyte reduction induced by hindlimb unloading: distinct mechanisms in the spleen and thymus. Cell Res 13: 465-471, 2003.

37. Dominguez-Gerpe L and Rey-Mendez M: Alterations induced by chronic stress in lymphocyte subsets of blood and primary and secondary immune organs of mice. BMC Immunol 2: 7, 2001.

38. Iwasaki M, Yu WG, Uekusa Y, et al: Differential IL-12 responsiveness of $\mathrm{T}$ cells but not of NK cells from tumorbearing mice in IL-12-responsive versus -unresponsive tumor models. Int Immunol 12: 701-709, 2000.

39. Gao P, Uekusa Y, Nakajima C, et al: Tumor vaccination that enhances antitumor T-cell responses does not inhibit the growth of established tumors even in combination with interleukin-12 treatment: the importance of inducing intratumoral T-cell migration. J Immunother 23: 643-653, 2000.

40. Monzavi-Karbassi B, Cunto-Amesty G, Luo P, Lees A and Kieber-Emmons T: Immunological characterization of peptide mimetics of carbohydrate antigens in vaccine design strategies. Biologicals 29: 249-257, 2001.

41. Dray S and Mokyr MB: Cyclophosphamide and melphalan as immunopotentiating agents in cancer therapy. Med Oncol Tumor Pharmacother 6: 77-85, 1989.

42. Li L, Okino T, Sugie T, et al: Cyclophosphamide given after active specific immunization augments antitumor immunity by modulation of Th1 commitment of $\mathrm{CD}^{+} \mathrm{T}$ cells. J Surg Oncol 67: 221-227, 1998.
43. Loeffler M, Kruger JA and Reisfeld RA: Immunostimulatory effects of low-dose cyclophosphamide are controlled by inducible nitric oxide synthase. Cancer Res 65: 5027-5030, 2005.

44. Ghiringhelli F, Larmonier N, Schmitt E, et al: $\mathrm{CD} 4{ }^{+} \mathrm{CD} 25^{+}$ regulatory $\mathrm{T}$ cells suppress tumor immunity but are sensitive to cyclophosphamide which allows immunotherapy of established tumors to be curative. Eur J Immunol 34: 336-344, 2004.

45. Ikezawa Y, Nakazawa M, Tamura C, Takahashi K, Minami M and Ikezawa Z: Cyclophosphamide decreases the number, percentage and the function of $\mathrm{CD} 25^{+} \mathrm{CD} 4^{+}$regulatory $\mathrm{T}$ cells, which suppress induction of contact hypersensitivity. J Dermatol Sci 39: 105-112, 2005.

46. Annacker O, Pimenta-Araujo R, Burlen-Defranoux O, Barbosa T, Cumano A and Bandeira A: $\mathrm{CD} 25^{+} \mathrm{CD} 4^{+} \mathrm{T}$-cells regulate the expansion of peripheral CD4 T-cells through the production of IL-10. J Immunol 166: 3008-3018, 2001.

47. Annacker O, Burlen-Defranoux O, Pimenta-Araujo R, Cumano A and Bandeira A: Regulatory CD4 T-cells control the size of the peripheral activated/memory CD4 T-cell compartment. J Immunol 164: 3573-3580, 2000.

48. Almeida ARM, Legrand N, Papiernik M and Freitas AA: Homeostasis of peripheral $\mathrm{CD}^{+}{ }^{+} \mathrm{T}$ cells: IL-2R(alpha) and IL-2 shape a population of regulatory cells that controls $\mathrm{CD}^{+} \mathrm{T}$ cell numbers. J Immunol 169: 4850-4860, 2002.

49. Uenaka A and Nakayama E: Murine leukemia RL male 1 and sarcoma Meth A antigens recognized by cytotoxic T lymphocytes (CTL). Cancer Sci 94: 931-936, 2003.

50. Ercolini AM, Ladle BH, Manning EA, et al: Recruitment of latent pools of high-avidity CD8(+) T cells to the antitumor immune response. J Exp Med 201: 1591-1602, 2005. 